\title{
Proliferation, migration and differentiation potential of human mesenchymal progenitor cells derived from osteoarthritic subchondral cancellous bone
}

\author{
Krüger JP ${ }^{1 \#}$, Enz $\mathrm{A}^{2 \#}$, Hondke $\mathrm{S}^{1}$, Wichelhaus $\mathrm{A}^{2}$, Endres $\mathrm{M}^{1}$, Mittlmeier $\mathrm{T}^{2}$
}

\begin{abstract}
Background: For regenerative therapies in the orthopedic field, one prerequisite for therapeutic success in the treatment of cartilage defects is the potential of body's own cells to migrate, proliferate and differentiate into functional cells. While this has been demonstrated for mesenchymal stem and progenitor cells (MPC) from healthy tissue sources, the potential of cells from degenerative conditions is unclear. In this study the regenerative potential of MPC derived from subchondral cancellous bone with diagnosed osteoarthritis is evaluated in vitro.

Methods: OaMPC isolated from bone chips of three individual patients with Kellgren grade 3 osteoarthritis were characterized by analysis of cell surface antigen pattern. Cell proliferation was evaluated by doubling time and population doubling rate. Cell migration was assessed using a multi-well migration assay. Multi-lineage potential was evaluated by histological staining of adipogenic, osteogenic and chondrogenic markers. In addition, chondrogenic differentiation was verified by qPCR.

Results: OaMPC showed a stable proliferation and a typical surface antigen pattern known from mesenchymal stem cells. Cell migration of oaMPC can be induced by human blood serum. OaMPC were capable of adipogenic, osteogenic and chondrogenic differentiation comparable to MPC derived from healthy conditions.

Conclusion: OaMPC derived from knee joints affected by osteoarthritic conditions showed regeneration potential regarding migration, proliferation and chondrogenic differentiation. This suggests that oaMPC are able to contribute to cartilage repair tissue formation.
\end{abstract}

Key Words: Osteoarthritis, Stem cells, Cartilage, Migration, Differentiation

\section{Introduction}

To date, the self-healing capacity of the human body is one of the most important sources for regenerative medicine. The aim is to rehabilitate malfunctioned cells and to regenerate parts of tissues or organs. A new generation of cell-free scaffolds, aiming at focal traumatic cartilage defects, should support the body's selfrenewal. Articular cartilage is frequently damaged as a result of trauma and degenerative joint diseases. Different cartilage regeneration strategies and techniques are used in today's clinical practice. Simple and often used techniques are the abrasion technique and the microfracture technique where mesenchymal stem and progenitor cells are used for regeneration approaches. Other regenerative strategies are the osteochondral transfer and the autologous chondrocyte implantation ${ }^{[1]}$. One concept among others is based on the well-known microfracture technique combined with an implant composed of e.g., resorbable polyglycolic acid combined with hyaluronan and a blood derivate $^{[2]}$ to cover the cartilage defect. A possible source of selfrenewal results from migrated progenitor cells since these cells are able to differentiate and synthesize a cartilage repair tissue ${ }^{[3]}$. The microfracture technique enables the influx of mesenchymal stem and progenitor cells into the cartilage defect ${ }^{[4]}$. Especially the migration, proliferation and differentiation potential of these cells are necessary for cartilage regeneration ${ }^{[3,5,6]}$ as the progenitor cells are attracted to the right place and support repair tissue formation. But, several pathological conditions such as rheumatoid arthritis or osteoarthritis (OA) may affect these cells. In a healthy joint the synovia covers the joint cavity and regulates the transport of nutrients and other molecules between the joint cavity and the adjacent tissue ${ }^{[7]}$. In joints affected by OA, the synovial membrane becomes hyperplastic due to the proliferation of genuine synovial cells such as synovial fibroblasts resulting in a massive infiltration of inflammatory cells ${ }^{[8]}$. Today, OA is attributed to diseases of articular cartilage. The current concept of OA involves the entire joint organ, including the articular cartilage, subchondral bones, ligaments, joint capsule, menisci and synovial membrane ${ }^{[9]}$. $\mathrm{OA}$ has always been classified as a non-inflammatory arthritis. However, increasing evidence has shown that inflammation occurs as cytokines and matrix metalloproteinases are released into the joint. This leads to the imbalance between inflammatory mediators and proteinase inhibitors compared to healthy individuals ${ }^{[10]}$. It is obvious that cartilage degradation and inflammatory surrounding of OA create a completely different environment, e.g., compared to traumatic defects. But, chondrogenesis of osteoarthritic chondrocytes proved that the chondrogenic capacity in vitro is not significantly affected by $\mathrm{OA}^{[11]}$. Furthermore, it was shown that synovial fluid from donors with $\mathrm{OA}$ does not have a negative influence on chondrogenesis ${ }^{[1,12]}$ and stimulates cell migration ${ }^{[13]}$ of mesenchymal progenitor cells (MPC). 
The regeneration potential of MPC from an osteoarthritic environment is still unknown. Therefore, the aim of this study was to characterize the proliferation, migration and differentiation potential of MPC derived from osteoarthritic subchondral cancellous bone, in order to prove if oaMPC are a suitable cell source for autologous matrixinduced chondrogenesis (aMIC).

\section{Method}

Isolation of osteoarthritic mesenchymal progenitor cells derived from osteoarthritic cortico-cancellous bone

Mesenchymal progenitor cells (oaMPC) were isolated from bone chips from knee joints of three individual OA patients (Table 1) with Kellgren-Lawrence grade 3 (Figure 1). In brief, the chips were cut in small fragments and washed with Hank's saline solution (Merck, Germany). Bone fragments were digested for 4 hours with 7,680U collagenase XI (Sigma-Aldrich, USA). Afterwards, the fragments were transferred into culture flasks (Primaria, Becton \& Dickinson, USA) and cultured with DMEM (Dulbecco's Modified Eagle Medium) containing 10\% human serum (German Red Cross, Germany), $100 \mathrm{U} / \mathrm{ml}$ penicillin, $100 \mu \mathrm{g} / \mathrm{ml}$ streptomycin, $200 \mathrm{nM}$ L-glutamine, $100 \mu \mathrm{g} / \mathrm{ml}$ gentamycin, $0.1 \mu \mathrm{g} / \mathrm{ml}$ amphotericin (all Biochrom) and 2ng/ml FGF-2 (Peprotech, Germany) at $37^{\circ} \mathrm{C}$ and $5 \%$ $\mathrm{CO}_{2}$. The medium was changed every 2-3 days. After the cells reached $80-90 \%$ confluence they were detached with trypsinEDTA/PBS $(1: 1 \mathrm{v} / \mathrm{v})$ (Merck) and seeded with a cell density of 8,000 cells $/ \mathrm{cm}^{2}$. The study was approved by the ethics committee of the Bavarian State Chamber of Medicine (\#12045).

\section{Migration Assay of oaMPC}

To characterize the migration potential of oaMPC ( $\mathrm{n}=3$ donors), a 96 multi-well migration assay (Neuro Probe, USA) was used. In brief, human serum in concentrations of 5\% and $10 \%$ diluted with DMEM containing $0.1 \%$ human serum (German Red Cross), 100U penicillin and $100 \mu \mathrm{g} / \mathrm{ml}$ streptomycin (Merck) was added into the lower wells of the multi-well plate and covered with a polycarbonate membrane (pore size of $8 \mu \mathrm{m}$ ). DMEM containing $0.1 \%$ human serum served as control. In the upper compartment of each well 30,000 oaMPC in DMEM containing $0.1 \%$ human serum was given. The assay was performed in triplicates. The assay was incubated at $37^{\circ} \mathrm{C}$ and $5 \%$ $\mathrm{CO}_{2}$ for 20 hours. Afterwards, the membrane was fixed with methanol/acetone $(1: 1 \mathrm{v} / \mathrm{v})$ for 5 minutes. Remaining oaMPC on top of the membrane were carefully wiped off and cells underneath the membrane were visualized using a Hemacolor staining kit (MerckMillipore, Germany). Migrated cells were enumerated microscopically by counting the number of stained cells in four representative fields using ImageJ software (National Institutes of Health, USA) and extrapolated to the complete well area.

\section{Growths kinetics of oaMPC}

To determine the growth kinetic of oaMPC, cells of each individual patient $(n=3)$ were seeded in T25 culture flasks with a density of
10,000 cells $/ \mathrm{cm}^{2}$ and cultivated for four days with DMEM containing $10 \%$ human serum, $100 \mathrm{U} / \mathrm{ml}$ penicillin, $100 \mu \mathrm{g} / \mathrm{ml}$ streptomycin, $200 \mathrm{nM}$ L-glutamine, $100 \mu \mathrm{g} / \mathrm{ml}$ gentamycin, $0.1 \mu \mathrm{g} / \mathrm{ml}$ amphotericin and $2 \mathrm{ng} / \mathrm{ml} \mathrm{FGF-2} \mathrm{at} 37^{\circ} \mathrm{C}$ and $5 \% \mathrm{CO}_{2}$. After four days of cultivation, vital cells were detached and counted with trypan blue and seeded again with the same cell density of 10,000 cells $/ \mathrm{cm}^{2}$. All in all, cells were cultivated from passage 1 to 7 . For each passage the proliferation factor was determined and the cell doubling time $\left(t_{d}\right)$ and the population doubling rate (n) were calculated as follows.

$$
\begin{aligned}
t_{d} & =\frac{\ln 2}{\text { specific growth rate }(\mu)} \\
n & =\frac{\log \frac{\text { initial cell number }}{\text { terminal cell number }}}{\log 2}
\end{aligned}
$$

Flow cytometric analysis of oaMPC

OaMPC $(250,000$ cells, passage 3$)$ of 3 donors were washed with $\mathrm{PBS} / 0.5 \%$ BSA and incubated with mouse anti-human conjugated antibody fluorescent CD14-phycoerythrin (PE), CD34-PE, CD44fluorescein isothiocyanate (FITC), CD45-FITC, CD73-PE, CD90FITC, CD105-FITC or CD166-PE (All Becton \& Dickinson, USA)

\section{Patient 1 Patient 2 Patient 3}
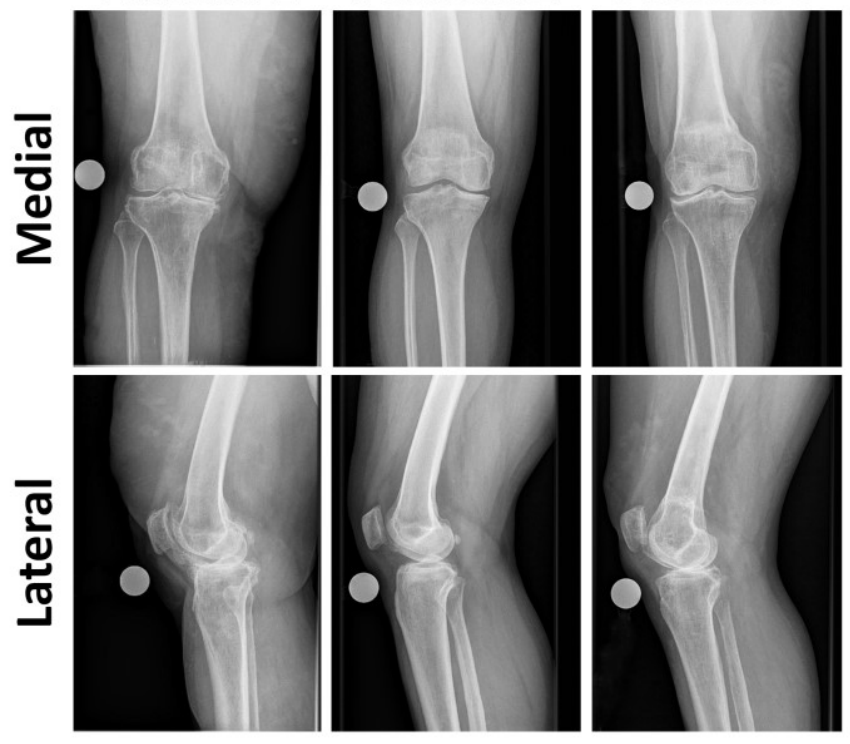

Figure 1: Medial and lateral x-ray images of the right knee form all three patients included in this study (all Kellgren and Lawrence score of 3).

Table 1: Patient characterization

\begin{tabular}{llll}
\hline & Patient 1 & Patient 2 & Patient 3 \\
\hline Age & $\begin{array}{l}\text { Gender } \\
\text { female }\end{array}$ & $\begin{array}{l}50 \text { years } \\
\text { female }\end{array}$ & $\begin{array}{l}60 \text { years } \\
\text { female }\end{array}$ \\
\hline Medical history & Primary gonarthrosis right knee & $\begin{array}{l}\text { Primary gonarthrosis right } \\
\text { knee }\end{array}$ & Primary gonarthrosis right knee \\
& Hypertrophic & Hypertrophic & Hypertrophic \\
Synovia & Total endoprosthesis of the knee & $\begin{array}{l}\text { Total endoprosthesis of the } \\
\text { knee joint }\end{array}$ & Total endoprosthesis of the knee \\
Knee replacement & joint & $\mathbf{3}$ & $\mathbf{3}$ \\
\hline Kellgren-Lawrence & $\mathbf{3}$ & & \\
\hline
\end{tabular}


For 15 minutes on ice. After incubation oaMPC were washed by adding $1 \mathrm{ml}$ buffer (PBS with $0.5 \%$ BSA), centrifuged at $400 \mathrm{xg}$ for 10 minutes at $4^{\circ} \mathrm{C}$ and resuspended in $500 \mu \mathrm{L}$ buffer. The analyses were performed using FACS Calibur (Becton \& Dickinson). Dead cells were excluded from analyses using propidium iodide (PI) and CD34 stained cells were used as isotype control.

Differentiation of oaMPC along adipogenic, osteogenic and chondrogenic lineage

To show multi-lineage differentiation potential of oaMPC, cells of 3 individual patients were differentiated into adipogenic, osteogenic and chondrogenic lineage.

For adipogenic and osteogenic differentiation oaMPC (passage 3) were plated with a density of 10,000 cells/well in 6-well cell culture plates (Becton \& Dickinson). To induce adipogenic differentiation oaMPC were stimulated with DMEM (Merck) containing 10\% human serum (German Red Cross), $100 \mathrm{U} / \mathrm{ml}$ penicillin and $100 \mu \mathrm{g} / \mathrm{ml}$ streptomycin (all Merck), $1 \mu \mathrm{M}$ dexamethasone, $0.2 \mathrm{mM}$ indomethacin, 0.5mM 3-isobutyl-1-methyl-xanthine (IBMX) (all Sigma-Aldrich) and $0.01 \mathrm{mg} / \mathrm{ml}$ insulin (Novo Nordisk, Denmark) for 21 days. In this process, oaMPC were stimulated for 3 days alternating with a nonstimulated period of 2 days. Non-stimulated controls were cultured without dexamethasone, indomethacin, IBMX and insulin.

For osteogenic differentiation, confluent oaMPC cultures were stimulated with DMEM containing $10 \%$ human serum, $100 \mathrm{U} / \mathrm{ml}$ penicillin, $100 \mu \mathrm{g} / \mathrm{ml}$ streptomycin, $0.01 \mu \mathrm{M}$ dexamethasone, $0.05 \mathrm{mM}$ ascorbic acid-2-phosphate (Sigma-Aldrich) and $10 \mathrm{mM}$ $\beta$-glycerolphosphate (Sigma-Aldrich) for 28 days. Non-stimulated controls were cultured without dexamethasone, ascorbic acid-2phosphate and $\beta$-glycerolphosphate.

For chondrogenic differentiation high-density pellet cultures $(250,000$ oaMPC per pellet) were formed by centrifugation at $130 \mathrm{xg}$ for 10 minutes. Chondrogenesis was induced by adding $10 \mathrm{ng} / \mathrm{ml}$ transforming growth factor-beta3 (TGFB3) (Peprotech) to DMEM (high glucose) containing $100 \mathrm{U} / \mathrm{ml}$ of penicillin and $100 \mu \mathrm{g} / \mathrm{ml}$ streptomycin (all Merck), 1\% (v/v) insulin-transferrin-sodium selenite (ITS+1), $0.1 \mu \mathrm{M}$ dexamethasone, $1 \mathrm{mM}$ sodium pyruvate, $0.17 \mathrm{mM}$ ascorbic acid-2-phosphate, $0.35 \mathrm{mM}$ L-proline (all Sigma-Aldrich). Non-stimulated controls were cultured without TGFB3. The pellets were cultivated for 21 days and the medium was changed every 2-3 days.

\section{Histological and immunohistochemical staining of oaMPC}

Intracellular lipid vacuoles in adipogenic cultures were visualized using Oil Red O staining (Sigma-Aldrich). Osteogenic differentiation was detected by staining of mineralized matrix components according to von Kossa. For detection of extracellular matrix deposition indicating chondrogenic differentiation, cryosections $(6 \mu \mathrm{m} ; \mathrm{n}=3$ cell pellets, $n=3$ sections per cell pellet for $n=3$ donor) were prepared and proteoglycan synthesis was visualized by alcian blue and safranin $\mathrm{O}$ staining. Collagen type II formation was visualized by incubation with rabbit anti-human type II collagen antibody (Acris, Germany) for 40 min. Colorimetrical detection was performed using 3-amino-9ethylcarbazole (EnVision++; Dako, Denmark) following a counterstaining with hematoxylin (Merck-Millipore).

Quantitative histomorphometric analysis was performed using the Adobe Photoshop Software CS6 as described ${ }^{[14]}$. In brief, a standard color was defined that represents the particular color of the specific staining. The tools "magic wand" and "select similar" were used to select areas of that particular color. The amount of stained pixels in relation to the total amount of pixels of the section gave the percentage of the positively stained area for proteoglycans or collagen type II, respectively.

\section{Gene expression analysis of oaMPC}

To evaluate the gene expression of typical chondrogenic marker genes, total RNA from 20 oaMPC pellet cultures per group was isolated at day 21 as described previously ${ }^{[15]}$. Subsequently, $3 \mu \mathrm{g}$ of total RNA was reversely transcribed with the iScript cDNA Synthesis Kit according to the manufacturer's instructions (BioRad, Germany). The relative expression level of the housekeeping gene glyceraldehyde-3-phosphate dehydrogenase (GAPDH) was used to normalize the samples. Real-time RT-PCR using i-Cycler PCR System (BioRad) was performed with $1 \mu \mathrm{L}$ of each cDNA sample in triplicates using the SYBR green PCR Core Kit (Applied Biosystems, USA). Relative quantification of gene expression was performed and is given as percentage of the GAPDH product.

\section{Statistical analysis}

Statistical analysis was performed using SigmaStat 3.5 (Systat Software GmbH, Germany). The Anderson-Darling test was used to determine normal distribution of data. Subsequently, one way analysis of variance with the post hoc analysis according to the Student-Newman-Keuls-Test was used. P-values less than 0.05 were considered statistically significant.

\section{Results}

\section{Growths kinetics of oaMPC}

OaMPC of 3 donors were expanded up to passage 7. Absolute cell counts from passage 1 to 7 were normalized to oaMPC growth of 250,000 initially seeded cells to calculate the cell doubling time $\left(t_{d}\right)$ and the population doubling rate $(\mathrm{n})$. The cell doubling time ranged from 3.67 to 3.99 days, and the population doubling rate was $8.09 \pm 0.57$.

\section{Migration potential of oaMPC}

The migration assay was performed to determine the migration potential of oaMPC stimulated with human serum (HS). In the presence of $0.1 \%$ HS 2,100 up to 2,500 oaMPC migrated through the pores of the membrane (Figure 2). Stimulation of oaMPC with 5\% HS resulted in a migration of 7,500 up to 10,300 cells and $10 \% \mathrm{HS}$ stimulated the migration of 6,300 up to 10,400 cells (Figure 2). Compared to oaMPC stimulated with $0.1 \% \mathrm{HS}$, the number of migrated cells was significantly increased $\left({ }^{*} \mathrm{p} \leq 0.0004\right)$ by stimulation with $5 \%$ and $10 \%$ HS for all 3 donors (Figure 2).

\section{Cell surface antigen pattern of oaMPC}

The immuno-phenotype of oaMPC of 3 patients was determined by flowcytometrical analyses (Figure 3). All oaMPC $(n=3)$, which were derived from osteoarthritic cancellous bone chips, were homogenously positive for CD44 (mean $99.96 \% \pm 0.0003 \%$ ), CD73 (mean 99.88\% $\pm 0.0006 \%$ ), CD90 (mean 99.52\% $\pm 0.006 \%$ ), CD105 (mean 90.35\% $\pm 0.04 \%$ ) and CD166 (mean 99.29\% $\pm 0.004 \%$ ). Additionally, oaMPC did not present CD14 (mean $0.02 \% \pm$ $0.0002 \%$ ), CD34 (mean $0.01 \% \pm 0.0001 \%$ ) and CD45 (mean $0.23 \%$ $\pm 0.004 \%$ ) (Table 2). 


\section{Patient 1 Patient 2 Patient 3}
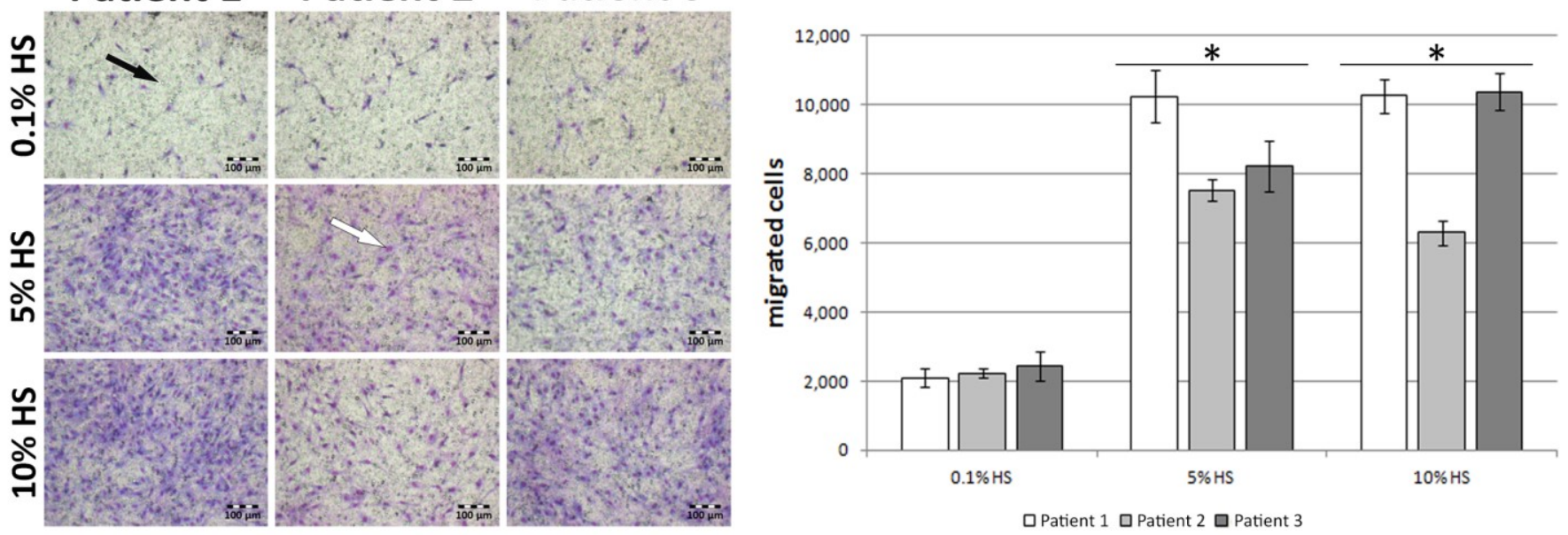

Figure 2: Medium with $5 \%$ or $10 \%$ human serum (HS) significantly $\left({ }^{*} p \leq 0.0004\right)$ stimulated the migration of osteoarthritic mesenchymal progenitor cells (black arrow indicates a membrane pore and white arrow indicates a migrated cell) compared to $0.1 \%$ HS. The bars show the mean ( $n=3$ triplicates) and the $95 \%$ CI.

Table 2: Flowcytometric analysis of oaMPC

\begin{tabular}{lllllllll}
\hline & CD14 & CD34 & CD44 & CD45 & CD73 & CD90 & CD105 & CD166 \\
\hline Patient 1 & $0.01 \%$ & $0.01 \%$ & $99.98 \%$ & $0.01 \%$ & $99.90 \%$ & $98.83 \%$ & $88.29 \%$ & $98.83 \%$ \\
Patient 2 & $0.01 \%$ & $0.01 \%$ & $99.93 \%$ & $0.01 \%$ & $99.81 \%$ & $99.97 \%$ & $87.63 \%$ & $99.71 \%$ \\
Patient 3 & $0.05 \%$ & $0.00 \%$ & $99.99 \%$ & $0.66 \%$ & $99.93 \%$ & $99.77 \%$ & $95.14 \%$ & $99.35 \%$ \\
\hline
\end{tabular}
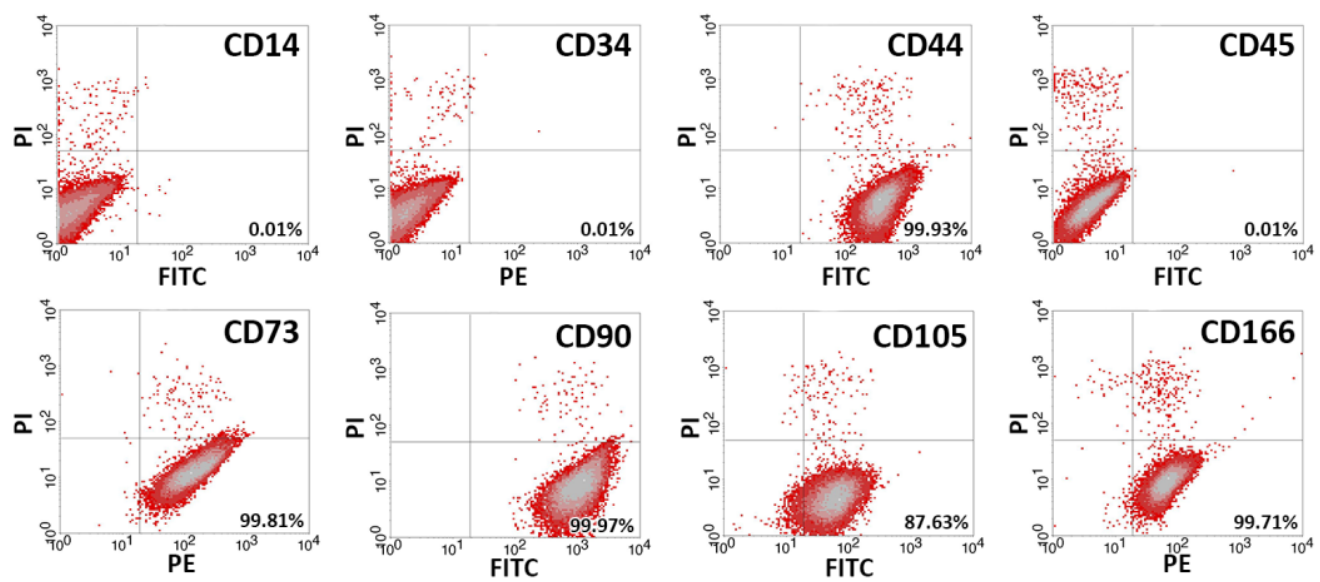

Figure 3: Flowcytometrical analyses of cell surface antigen pattern for one representative patient.

\section{Differentiation potential of oaMPC}

The potential of oaMPC to undergo adipogenic and osteogenic differentiation was documented by the visualization of lipid vesicles by Oil Red $\mathrm{O}$ staining and of matrix mineralization by von Kossa staining. At day 21, Oil Red $\mathrm{O}$ staining showed that lipid-filled vesicles were presented in oaMPC stimulated with adipogenic supplements. In non-induced oaMPC no lipid vesicles were detectable (Figure 4). At day 28, von Kossa staining showed a brown stained matrix in oaMPC stimulated with osteogenic supplements with only slight staining in the non-induced controls (Figure 4).

After 28 days of culture in high-density pellets, oaMPC showed a chondrogenic differentiation upon stimulation with TGFB3, while no signs of chondrogenesis were observed in non-induced controls (Figure 5). Proteoglycans as assessed by alcian blue staining were evident in pellets stimulated with TGFB3 and weakly in non-induced oaMPC pellets. Safranin O stained sulfated proteoglycans were detectable in oaMPC pellets induced with TGFB3, while non-induced samples were negative. Collagen type II was found in pellets cultured with TGFB3 but not in non-induced oaMPC pellets.

Histomorphometric quantification $(\mathrm{n}=9 ; 3$ sections per patient) (Figure 5) showed a significant $\left({ }^{*} p<0.0003\right)$ increase of proteoglycans visualized by alcian blue staining in oaMPC pellet cultures induced with TGFB3 from day 7 (mean $42.1 \% \pm 12.1 \%$ ) to day 21 (mean $74.3 \% \pm 10.2 \%$ ). The non-induced oaMPC pellet cultures showed an increasing concentration of proteoglycans (but not significant) from day 7 (mean $10.5 \% \pm 5.1 \%$ ) to day 21 (mean $31.0 \% \pm 13.0 \%$ ). Quantification of sulfated proteoglycans (safranin $\mathrm{O}$ staining) showed a significant $\left({ }^{*} \mathrm{p}<0.0002\right)$ increase of proteoglycan-rich areas in pellets stimulated with TGFB3 from day 7 (mean $11.7 \% \pm 4.2 \%$ ) to day 21 (mean $77.4 \% \pm 4.9 \%$ ). In contrast, non-induced oaMPC pellet cultures showed almost no proteoglycanrich areas at day 7, day 14 and day 21 (mean $0.01 \%$ to $0.1 \%$ ). Collagen type II was present and significantly $\left(\mathrm{p}^{*}<0.0002\right)$ increased in oaMPC pellet cultures induced with TGFB3 from day 14 (mean $46.8 \% \pm 8.2 \%$ ) to day 21 (mean $59.9 \% \pm 6.3 \%$ ) compared to noninduced oaMPC pellet cultures from day 14 (mean $1.9 \% \pm 1.1 \%$ ) to day 21 (mean $2.7 \% \pm 1.1 \%)$. 


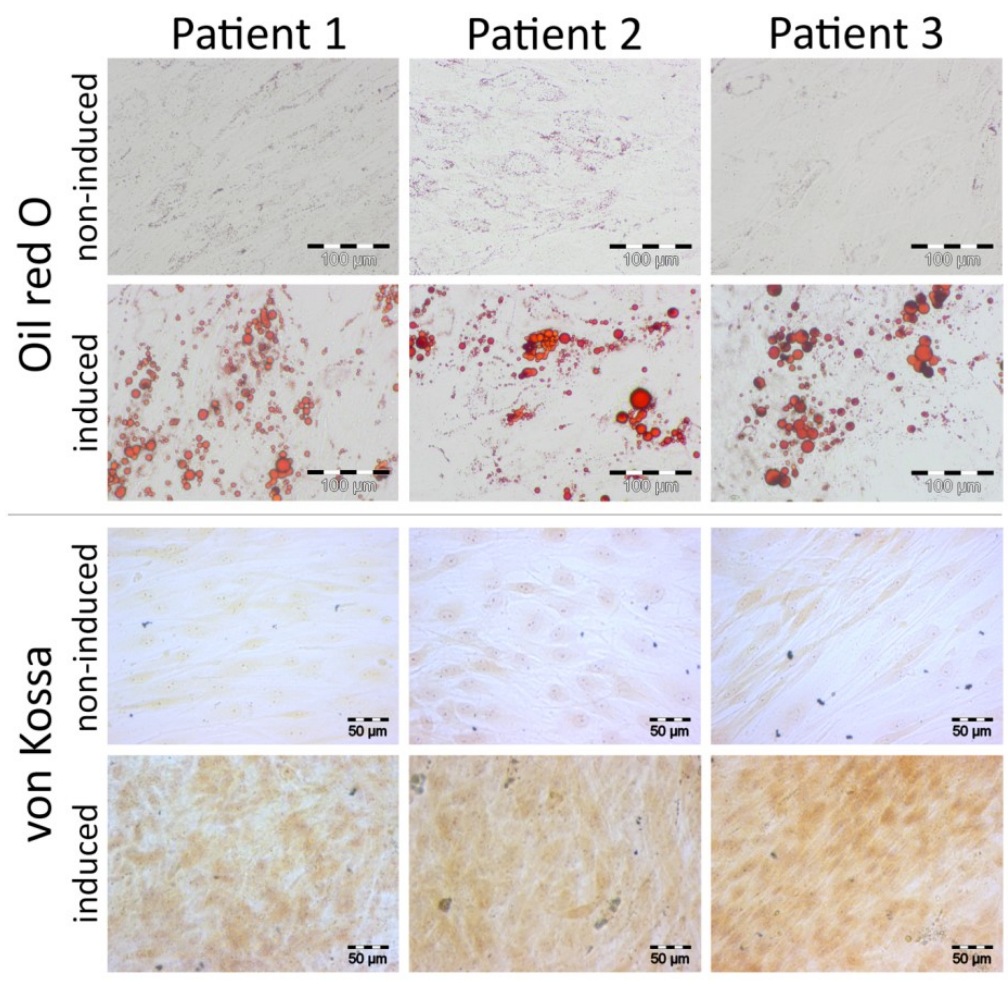

Figure 4: Oil Red O staining showed the presence of lipid vacuoles in adipogenic induced osteoarthritic mesenchymal progenitor cells (oaMPC) at day 21. Von Kossa staining of matrix mineralization showed a brown stained matrix in osteogenic induced oaMPC at day 28.

Figure 5: Osteoarthritic mesenchymal progenitor cells (oaMPC) induced with TGFB3 showed a significant $\left({ }^{*} p<0.0003\right) \quad$ increased formation of proteoglycans and collagen type II after 21 days compared to non-induced oaMPC. The bars show the mean ( $n=9 ; \quad 3$ sections/pellet/donor) and the $95 \% \mathrm{CI}$.

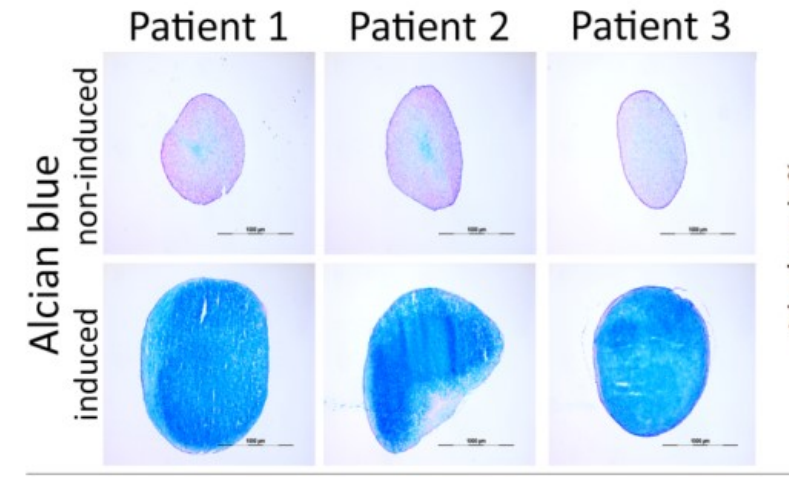

Histomorphometric analysis
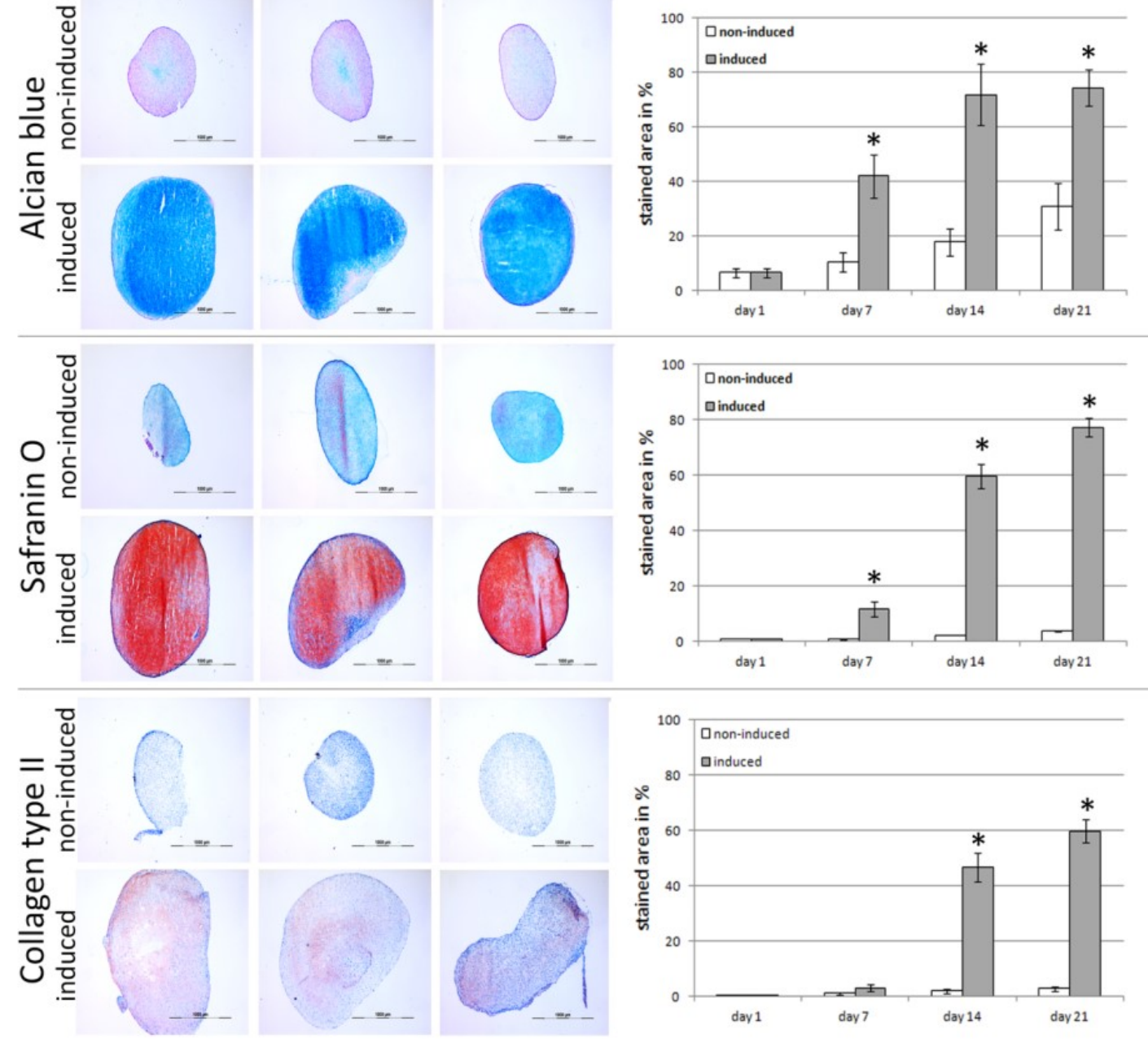
Linkprotein

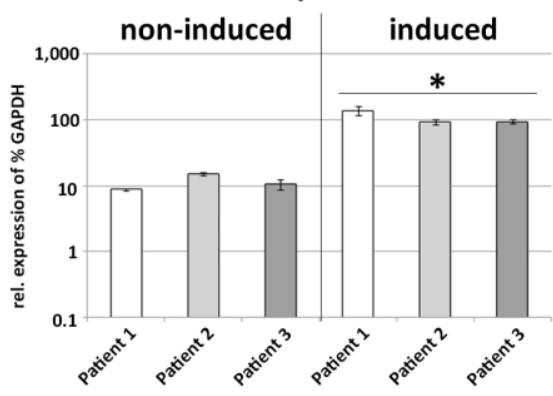

Collagen type I

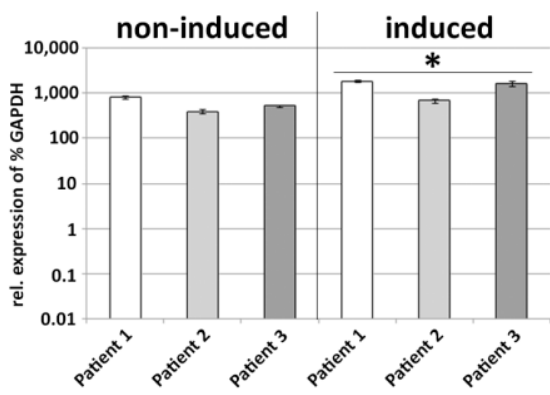

Collagen type $X$

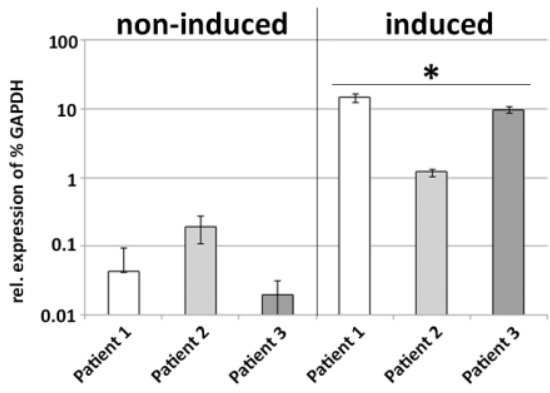

COMP

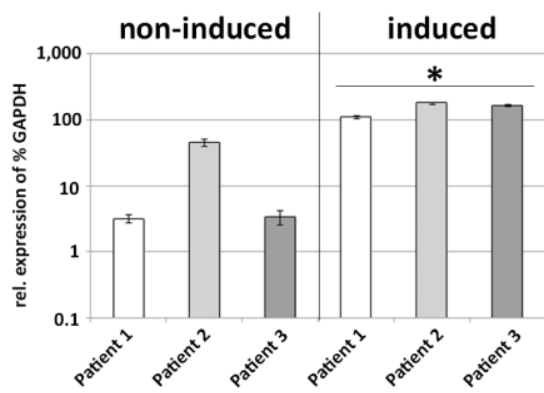

Collagen type II

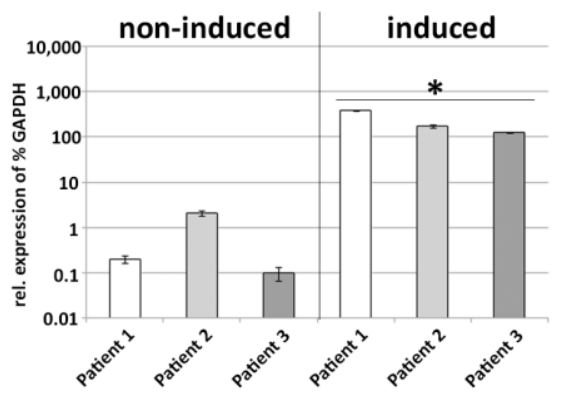

Osteocalcin

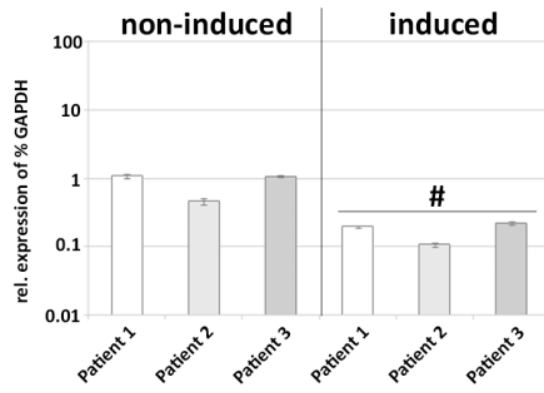

Aggrecan

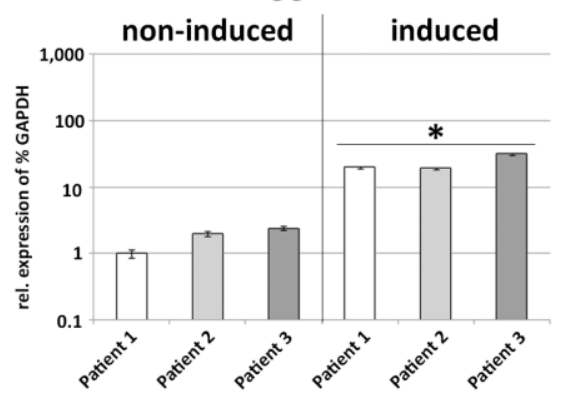

Collagen type IX

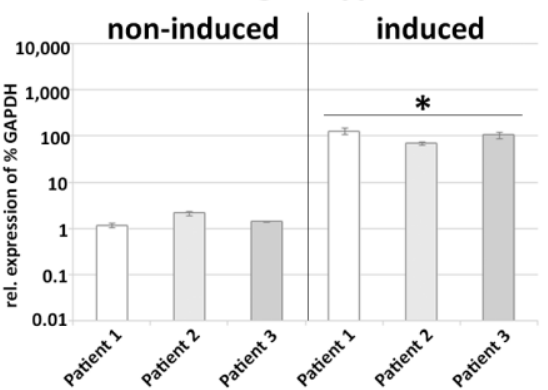

FABP4

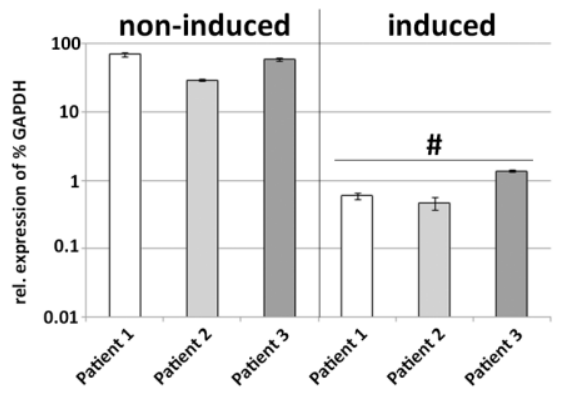

Figure 6: Chondrogenic development of osteoarthritic mesenchymal progenitor cells (oaMPC) was analyzed by gene expression analysis of typical chondrogenic marker genes. The bars show the mean ( $n=3$ triplicates) and the 95\% CI. Significantly $(p<0.0012){ }^{*}$ induced or ${ }^{\#}$ decreased gene expression compared to non-induced oaMPC. COMP - cartilage oligomeric matrix protein, FABP4 - fatty acid binding protein-4

\section{Gene expression analysis of oaMPC}

To confirm chondrogenic differentiation of oaMPC ( $\mathrm{n}=3)$, semiquantitative gene expression of typical cartilage marker were analyzed at day 21 (Figure 6). Treatment of oaMPC pellet cultures with TGFB3 significantly $\left({ }^{*} \mathrm{p}<0.0012\right)$ induced the expression of the chondrogenic marker genes link-protein (mean 100 times), cartilage oligomeric matrix protein (COMP, mean 10 times), aggrecan (mean 13 times), collagen type II (mean 230 times) and collagen type IX (mean 50 times), compared to non-induced oaMPC pellet cultures. Collagen type I, a gene which is more associated with fibrous tissue development showed an induction (mean 3 times). To evaluate osteogenic or adipogenic differentiation of oaMPC pellet cultures, gene expression analyses of selected osteogenic and adipogenic markers were performed at day 21. Pellet cultures of oaMPC induced with TGFB3 showed a significant $(* p<0.0002)$ increase of collagen type $\mathrm{X}$ (mean 85 times). In contrast, the gene expression level of osteocalcin was significantly decreased $(\# p<0.0003)$ in oaMPC cultures treated with TGFB3 (mean 5 times) compared to noninduced cultures. At day 21, oaMPC cultures induced with TGFB3 showed a significant $(\# p<0.0001)$ decrease in expression level of fatty acid binding protein-4 (FABP4, mean 65 times) compared to noninduced pellet cultures.

\section{Discussion}

In our study, we demonstrated that human osteoarthritic mesenchymal progenitor cells (oaMPC) have the ability to migrate, proliferate and differentiate along the adipogenic, osteogenic and chondrogenic lineage. OaMPC did not lose their migration potential in vitro in response to the osteoarthritic disorder. These findings are in line with previous experiments with mesenchymal stem and progenitor cells from healthy donors ${ }^{[5]}$. Additionally, the growth kinetics for oaMPC showed a stable proliferation and there were no differences between the respective population doubling times and population doubling rates of the three patients. In contrast, the population doubling rate of MPC without an inflammatory environment is twofold higher ${ }^{[16]}$. But, a reduced proliferation capacity is described for chondrocytes as well as for mesenchymal stem and progenitor cells from patients with osteoarthritis ${ }^{[17,18]}$. It is conceivable that the reduced proliferation capacity of oaMPC is a consequence of the osteoarthritic disorder. For verification of the progenitor cell character, oaMPC were analyzed by flowcytometrical analyses of typical surface antigen known from MPC. A change of phenotype or a less presentation of surface antigens is not mentionable in comparison to healthy $\mathrm{MPC}^{[1,16]}$. Furthermore, oaMPC were differentiated along the adipogenic, osteogenic and 
chondrogenic lineage. A previous study suggested a reduced adipogenic differentiation potential for osteoarthritic mesenchymal stem and progenitor cells from patients who received a total hip or total knee replacement ${ }^{[18]}$, which could not be confirmed by our results. Osteogenic induced oaMPC showed a less calcified matrix by von Kossa staining, but it is not possible to conclude that the osteoarthritic disorder is responsible for the insufficient mineralization of the extracellular matrix. Other differentiation studies with MPC demonstrated various results showing insufficient mineralization of the formed matrix after 28 days of cultivation ${ }^{[3]}$ as well as a highly calcified matrix after 18 days of cultivation ${ }^{[1]}$. Therefore, it is not clear if the insufficiently calcified matrix is a result of the influence of the degenerative disease or a normal appearance during osteogenesis of MPC/oaMPC. Sections of chondrogenic induced high-density oaMPC pellet cultures demonstrated a proteoglycan-rich extracellular matrix. In contrast to the uniformly stained TGFB3 induced oaMPC pellets, the noninduced controls showed marginal proteoglycan-rich matrix, which indicates a TGFB3-mediated chondrogenesis. Nevertheless, in noninduced controls marginal deposition of proteoglycans is evident that can be explained by the influence of the $3 \mathrm{D}$ arrangement ${ }^{[19]}$ which is also known to induce or at least support chondrogenesis. The results on protein level are in line with the results obtained by gene expression analysis of typical chondrogenic marker genes. There are no notable differences of protein or gene expression results compared to $\mathrm{MPC}^{[3]}$. However, a significant increase of collagen type I expression by oaMPC compared to MPC without an inflammatory environment could be noticed ${ }^{[3]}$. That may lead to the assumption that the chondrogenic induced oaMPC pellet cultures form fibrocartilaginous tissue or a hybrid of hyaline and fibrous cartilage depending on the co-presence of collagen type $\mathrm{II}^{[19,20]}$. However, our current study has some limitations. This in vitro study does not reflect the in vivo situation and cannot predict effects or activities of an inflammatory environment in vivo. Due to limited donor tissue availability, we included only female tissue donors of the same age group in this study. However, it is described that two times more females are affected by gonarthrosis than $\operatorname{men}^{[21]}$. Possible gender specific differences in the chondrogenic regeneration potential have to be analyzed in clinical studies and cannot be predicted from our results. Taking into account that the in vivo environment of cartilage in osteoarthritis (OA) is affected by several pathological conditions such as a hyperplastic synovial membrane and a massive infiltration of inflammatory cells, the clinical outcome of regenerative approaches (e.g., autologous matrix induced chondrogenesis (aMIC)) is not predictable. Under normal conditions the cartilage matrix is subjected to a dynamic remodeling process in which low levels of degradative and synthesizing enzyme activities are balanced, so that natural turnover of cartilage is maintained ${ }^{[22]}$. In advanced OA, cartilage matrix degrading enzymes are overexpressed shifting the balance to an increased degradation that might result in the loss of collagen and proteoglycans from the cartilage matrix ${ }^{[1]}$. First case series showed good results in patients suffering from beginning articular degeneration and early degenerative cartilage defects one and five years after microfracture and aMIC procedure using a resorbable polyglycolic acid (PGA) implant ${ }^{[23,24]}$. From our results we suggest that oaMPC have the potential to undergo chondrogenic differentiation and might be useful as autologous source for regenerative therapies, but the etiology of inflammatory environment or abnormal mechanics need to be eliminated before ${ }^{[25]}$. Nevertheless, clinical studies need to be undertaken to show whether oaMPC are able to develop sufficient repair tissue for cartilage defect treatment.
In conclusion, it is possible to say that MPC from patients with osteoarthritis present a comparable phenotypic antigen pattern and the potential of migration, proliferation, and multi-lineage differentiation along chondrogenesis, adipogenesis and osteogenesis to MPC from healthy patients.

\section{References}

1. Krüger JP, Endres M, Neumann K, Stuhlmuller B, Morawietz L, Haupl T, Kaps C. Chondrogenic differentiation of human subchondral progenitor cells is affected by synovial fluid from donors with osteoarthritis or rheumatoid arthritis. J Orthop Surg Res. 2012;7:10.

2. Erggelet C, Neumann K, Endres M, Haberstroh K, Sittinger M, Kaps C. Regeneration of ovine articular cartilage defects by cellfree polymer-based implants. Biomaterials. 2007;28(36):55705580 .

3. Neumann K, Dehne T, Endres M, Erggelet C, Kaps C, Ringe J, Sittinger M. Chondrogenic differentiation capacity of human mesenchymal progenitor cells derived from subchondral corticospongious bone. J Orthop Res. 2008;26(11):1449-1456.

4. Steadman JR, Briggs KK, Rodrigo JJ, Kocher MS, Gill TJ, Rodkey WG. Outcomes of microfracture for traumatic chondral defects of the knee: average 11-year follow-up. Arthroscopy. 2003;19(5):477-484.

5. Kruger JP, Hondke S, Endres M, Pruss A, Siclari A, Kaps C. Human platelet-rich plasma stimulates migration and chondrogenic differentiation of human subchondral progenitor cells. J Orthop Res. 2012;30(6):845-852.

6. Kreuz PC, Kruger JP, Metzlaff S, Freymann U, Endres M, Pruss A, Petersen W, Kaps C. Platelet-Rich Plasma Preparation Types Show Impact on Chondrogenic Differentiation, Migration, and Proliferation of Human Subchondral Mesenchymal Progenitor Cells. Arthroscopy. 2015 ;31(10):1951-1961.

7. Schmid FR, Ogata RI. The composition and examination of synovial fluid. J Prosthet Dent. 1967;18(5):449-457.

8. Kraan MC, Versendaal H, Jonker M, Bresnihan B, Post WJ, t Hart BA, Breedveld FC, Tak PP. Asymptomatic synovitis precedes clinically manifest arthritis. Arthritis Rheum. 1998;41(8):14811488.

9. Braun HJ, Gold GE. Diagnosis of osteoarthritis: imaging. Bone. 2012;51(2):278-288.

10. Murphy G, Knäuper V, Atkinson S, Butler G, English W, Hutton M, Stracke J, Clark I. Matrix metalloproteinases in arthritic disease. Arthritis Res. 2002;4 Suppl 3:S39-49.

11. Dehne $T$, Karlsson C, Ringe J, Sittinger M, Lindahl A. Chondrogenic differentiation potential of osteoarthritic chondrocytes and their possible use in matrix-associated autologous chondrocyte transplantation. Arthritis Res Ther. 2009;11(5):R133.

12. Krüger JP, Endres M, Neumann K, Haupl T, Erggelet C, Kaps C. Chondrogenic differentiation of human subchondral progenitor cells is impaired by rheumatoid arthritis synovial fluid. J Orthop Res. 2010;28(6):819-827.

13. Endres M, Neumann K, Haupl T, Erggelet C, Ringe J, Sittinger M, Kaps C. Synovial fluid recruits human mesenchymal progenitors from subchondral spongious bone marrow. J Orthop Res. 2007;25(10):1299-1307.

14. Kruger JP, Machens I, Lahner M, Endres M, Kaps C. Initial boost release of transforming growth factor-beta3 and chondrogenesis by freeze-dried bioactive polymer scaffolds. Ann Biomed Eng. $2014 ; 42(12): 2562-2576$.

15. Chomczynski P. A reagent for the single-step simultaneous isolation of RNA, DNA and proteins from cell and tissue samples. Biotechniques. 1993;15(3):532-534, 536-537.

16. Tuli R, Tuli S, Nandi S, Wang ML, Alexander PG, Haleem-Smith H, Hozack WJ, Manner PA, Danielson KG, Tuan RS. Characterization of multipotential mesenchymal progenitor cells derived from human trabecular bone. Stem Cells. 2003;21(6):681693. 
17. Aigner T, Kurz B, Fukui N, Sandell L. Roles of chondrocytes in the pathogenesis of osteoarthritis. Curr Opin Rheumatol. 2002;14(5):578-584.

18. Murphy JM, Dixon K, Beck S, Fabian D, Feldman A, Barry F. Reduced chondrogenic and adipogenic activity of mesenchymal stem cells from patients with advanced osteoarthritis. Arthritis Rheum. 2002;46(3):704-713.

19. Richter W. Mesenchymal stem cells and cartilage in situ regeneration. J Intern Med. 2009;266(4):390-405.

20. Kaul G, Cucchiarini M, Remberger K, Kohn D, Madry H. Failed cartilage repair for early osteoarthritis defects: a biochemical, histological and immunohistochemical analysis of the repair tissue after treatment with marrow-stimulation techniques. Knee Surg Sports Traumatol Arthrosc. 2012;20(11):2315-2324.
21. Glyn-Jones S, Palmer AJ, Agricola R, Price AJ, Vincent TL, Weinans H, Carr AJ. Osteoarthritis. Lancet. 2015;386(9991):376387.

22. Goldring MB, Tsuchimochi K, Ijiri K. The control of chondrogenesis. J Cell Biochem. 2006;97(1):33-44

23. Siclari A, Mascaro G, Gentili C, Cancedda R, Boux E. A cell-free scaffold-based cartilage repair provides improved function hyaline-like repair at one year. Clin Orthop Relat Res. 2012 ;470(3):910-919.

24. Siclari A, Mascaro G, Kaps C, Boux E. A 5-year follow-up after cartilage repair in the knee using a platelet-rich plasma-immersed polymer-based implant. Open Orthop J. 2014;8:346-354.

25. Hogenmiller MS, Lozada CJ. An update on osteoarthritis therapeutics. Curr Opin Rheumatol. 2006;18(3):256-260.

\author{
Abbreviations \\ CI: $\quad$ Confidence interval \\ COMP: $\quad$ Cartilage oligomeric matrix protein \\ DMEM: $\quad$ Dulbecco's Modified Eagle Medium \\ FABP4: $\quad$ Fatty acid binding protein-4 \\ FITC: $\quad$ Fluorescein isothiocyanate \\ HS: Human serum \\ IBMX: 3-isobutyl-1-methyl-xanthine \\ ITS+1: $\quad$ Insulin-transferrin-sodium selenite \\ MPC: $\quad$ Mesenchymal progenitor cells \\ oaMPC: $\quad$ Mesenchymal progenitor cells derived from osteoarthritic tissue \\ PE: $\quad$ Phycoerythrin \\ PI: $\quad$ Propidium iodide \\ TGFB3: Transforming growth factor-beta3
}

\title{
Potential Conflicts of Interests
}

Authors Krüger, Hondke \& Endres are employees of TransTissue Technologies GmbH, Germany.

\section{Acknowledgements}

The authors thank Annina Wanzek and Samuel Vetterlein for their excellent technical assistance.

\section{Corresponding Author}

Michaela Endres, TransTissue Technologies GmbH Charitéplatz 1, 10117 Berlin, Germany; Email: michaela.endres@transtissue.com 